\title{
Effects of erythropoietin in improving function of spinal cord injury
}

Ali Meshkini ${ }^{1}$, Nasrin Pourhajshokr ${ }^{1}$, Firooz Salehpour ${ }^{1}$, Parviz SamadiMotlagh ${ }^{1}$, M.Taghi Imani Kh ${ }^{*}$

1. Department of Neurosurgery, NeurosciencesResearch Center ,Imam Reza Hospital, Faculty of Medicine, Tabriz University of Medical Sciences, Tabriz, Iran

2. Students' Research Committee, Tabriz University of Medical Science, Tabriz, Iran

\begin{abstract}
Introduction: Acute Spinal Cord Injury (ASCI) is one of the most common and important disorders in the field of neurosurgery. Progress achieved in relation to the care provided to repair spinal cord injuries have been taken from ancient times to the present day. Recently Neuroprotective therapies have attracted a lot of staff to approach the patient. Research scientists have shown that there is a possibility of recovery after spinal cord injury. Many pharmacological agents, Erythropoietin, in this field are used to reduce secondary damage after the primary insult and try to preserve nerve tissue. The aim of this study was to investigate the effect of Erythropoietin on sensory and motor status of patients with acute spinal cord injury. Materials and Methods: In this clinical trial, 60 patients with acute spinal cord injury in the Frankel classification, the category $A$ to $C$, and the selection of matched Frankel class into two groups A and B (each group consisted of 30 patients) was done. Group A underwent conventional treatment received methylprednisolone, were used erythropoietin and were compared after 4days,6months intervals in terms of complete and incomplete cord injury status with group B (that underwent only conventional treatment such as methylprednisolone). Results: In our study of two groups there were 16 patients with complete spinal cord injury and 44 patients were had incomplete $\mathrm{SCl}$. In the period of 4 days after the onset of the study, patients with complete $\mathrm{SCl}$ in case and control group did not recover. In Patients with incomplete spinal cord injury in case group 13 of 21 patients (62\%) and 2 of 23 patients (9\%) in the control group were cured. In the period of 6 months after the study, 2 of 8 patients $(25 \%)$ in case group with complete cord had recovery. In the control group of patients with complete spinal cord injury didn't have any recovery. Also in incomplete $\mathrm{SCl} 12$ of 19 patients (63\%) in case group and 5 of 21 patients (23\%) in the control group were cured. Conclusion: Based on the results obtained improvement in muscle strength and sensory classified according to Frankel in patients receiving erythropoietin in period between 4 days and 6 months can be seen that the difference significant with the control group $(P<0.0001)$. So Frankel class $B, C$ to $C-D-E$ Frankel grade was reached after recovery.
\end{abstract}

Keywords: Acute spinal cord injury; Classification Frankel; Erythropoietin; Methylprednisolone

\section{Corresponding author:}

Mohammad Taghi Imani

Prosthodontics Department, Dental School, Tabriz University of Medical Sciences, Tabriz, Iran

Email: imanitaghi@yahoo.com Mobile:09141049391

Receive date: 2015-10-11| Accept date: 2015-10-29| Publish date: 2015-12-01

DOI: 10.7575/aiac.abcmed.16.04.01.03

\section{A. I}




\section{Introduction}

Acute Spinal cord injury (ASCl)is among the most common and important disorders in the field of neurosurgery and causes sensory, motor, urinary tract impairment or a combination of these and in our country are consist a large proportion of patients that referred to trauma centers (1). Progress achieved in relation to the care provided to repair spinal cord injuries have been taken from ancient times to the present day (2) Research scientists have shown that there is a possibility of recovery after acute spinal cord injury (2). The classic two types management for these patients are: Conservative and surgical treatment, if necessary (3). Now methylprednisolone is only protocol treatment with part of the therapeutic benefit and associated dangerous side effects. Recently Neuroprotective therapies such as erythropoietin - Minocycline - progesterone etc., have attracted a lot of staff to approach the patients (1-4). Many pharmacological agents in fact, in this field are used to reduce secondary damage after the primary insult and try to preserve nerve tissue (5). Inflammation plays an important role in spinal cord injury. Although the use of anti-inflammatory such as prednisolone have the beneficial effect, but use of methylprednisolone have had the moderate beneficial effects.

For serious complications of spinal cord injury, use of erythropoietin to reduce inflammation and limit the neuronal apoptosis is benefit. Erythropoietin increases cell survival through inhibition of apoptosis. Erythropoietin has a direct impact on reducing inflammation and apoptosis addition can also improve vascular perfusion (6). Cytokine EPO has a strong protective effect against brain and spinal cord injury including transient brain ischemic and restores blood flow and improves spinal cord injury (7). Damage the nervous system stimulates proinflammatory cytokines and other molecules of complicated cascade that ultimately leads to necrosis -apoptosis neurons - oligodendrocytes and endothelial cells (8). Recent studies have shown that a general response in the brain and spinal cord injury in an increase in erythropoietin and receptors erythropoietin.

Erythropoietin important role in the CNS of mice devoid of the EPO-R study found a lack of EPO-R. In these mice, large amounts of apoptosis and neuronal precursor cells showed reduction. (14)

In this study there in Tabriz -Iran Department Biochemistry in 2010 was done on rat spinal cord injury showed that erythropoietin has properties antioxidant and reduce per oxidation lipid (11).

The aim of this study was to investigate the effect of Erythropoietin on sensory and motor status (Frankel) of patients with acute spinal cord injury.

\section{Materials and Methods}

In this clinical trial, 60 patients with acute spinal cord injury in the Frankel classification, the category $A$ to $C$, and the selection of matched Frankel class into two groups $A$ and $B$ (each group consisted of 30 patients) was done. Group A underwent conventional treatment received methylprednisolone, were used erythropoietin were compared after 4days,6months intervals in terms of complete and incomplete cord injury status with group $B$ (that underwent only conventional treatment such as methylprednisolone.)

The study included patients with acute spinal cord injury population in Center of Imam Reza hospital within a year.

Inclusion criteria:

- Acute spinal cord injury with Frankel A to C. 
- To the hospital during the first 8 hours of trauma.

- An informed consent to participate in the study.

- C1-T12 vertebral fractures spine.

Exclusion criteria:

- Patients with uncontrolled high blood pressure

- Patients with hypersensitivity to human albumin.

- Pregnancy and lactation.

- Erythroid leukemia.

- Patients with a history of thrombo embolic events.

- Patients with severe coronary artery, peripheral artery - Carotid or brain diseases.

- Brain injury patients with low GCS due to lack of accurate determination of the sensory and motor patients.

In all patients with traumatic spinal cord injury, spinal (thoracic-cervical) and damage neurological (complete or incomplete), in the admission stage of sensory-motor patients were classified according to Frankel. The protocol treatment for all patients in the guidelines of neurosurgery was performed as follows (6):

All patients who were taken to hospital with acute spinal cord injuries during the first 8 hours, methylprednisolone $33 \mathrm{mg} / \mathrm{kg}$ as a bolus intravenously within fifteen minutes infusion.

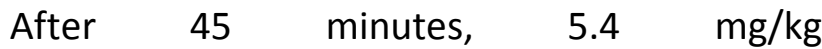
methylprednisolone infusion within 23-47 hours then patients can be divided into two groups, the first group (cases) in addition to methylprednisolone, after obtaining the consent of the patient, erythropoietin 500 units per kilogram of body weight by intravenous infusion in 3 divided doses given within 3 days(7).
(Recombinant human erythropoietin Alfa produced by recombinant DNA technology vial contains 165 amino acids and has a molecular weight of 34,000 Daltons. 2000 units in a vial and the construction of Iran's Exir Pharmaceuticals).

In the second group (control) additional treatment will not added other than methylprednisolone. Muscle strength and sensory level Frankel by category A-E in 4 days and discharge time and 6 months after trauma will be taken in both groups.

During the study, patients in the baseline period, 4 days and 6 months after baseline were examined. Before the trial begins by explaining all of the patients wrote consent. Patients are free to leave the study and not to participate in the study at any time of the study.

Data obtained by using descriptive statistics (Mean $\pm \mathrm{SE}$ ), frequency, percentage and mean difference test (T-Test, chi-Square Test) for independent samples for quantitative variables and chi-square test for qualitative variables and using the statistical software SPSS ${ }^{\text {тM }} 16$ review and statistical analysis was performed. The $P$ values less than 0.05 was considered statistically significant.

\section{Results}

Finally, 54 people (90\%) completed this study and $6(10 \%)$ patients died. Of the 60 patients, 45 patients (75\%) were male and 15 patients $(25 \%)$ were female $(p=0.6)$.

Of the 60 patients, 29 patients (48\%) with cervical spinal cord injury and 31 patients (52\%) thoracic spinal cord injury.

Check the status of recovery or non-recovery in spinal cord injury patients in two groups based on 4 days and 6 months after starting the study in Tables 1-4 and 2-4. 


\begin{tabular}{lr|lll}
\hline & & Case group & Control group & P value \\
\hline Cord injury Complete & $\begin{array}{r}\text { recovery } \\
\text { Non recovery }\end{array}$ & $9(\% 100)$ & $7(\% 100)$ & \\
\hline Cord injury Incomplete & recovery & $13(\% 62)$ & $2(\% 9)$ & $<0.0001$ \\
& Non recovery & $8(\% 38)$ & $21(\% 91)$ &
\end{tabular}

Table 1: Check the status of the recovery or non-recovery of both groups based on the type of injury four days after the beginning of the study

\begin{tabular}{r|ccc}
\hline & Case group & Control group & P value \\
\hline Complete cord injury & & & $<0.0001$ \\
Recovery & $2(\% 25)$ & 0 & \\
no recovery & $6(\% 75)$ & $6(\% 100)$ & \\
\hline Incomplete cord injury & & & $<0.0001$ \\
Recovery & $12(\% 63)$ & $5(\% 23)$ & \\
No recovery & $7(\% 37)$ & $16(\% 76)$ &
\end{tabular}

Table 2: Check the status of recovery or non-recovery in two groups based on the type of spinal cord injury patients 6 months after the beginning of the study

\section{Discussion}

Spinal trauma disease is the most significant injuries which can cripple a person for a lifetime, low quality of life, cost of care for patients and the short life of the individual. Health measurements with early treatment is important to improve the quality of life of patients. (8-9-10). Therefore, in this study the Neuroprotective effect of erythropoietin drug in acute spinal cord injury patients with severe sensory and motor dysfunction were studied.

Erythropoietinhormoneis a 165 amino acid glycoprotein that belongs to the cytokine type I family. Initially they believed that it only plays the role of erythropoietin erythropoiesis regulation of erythropoietin ability to inhibit apoptosis in erythroid cells, which is a result of the maturation of erythroid cells (11-21-22-2324).
In compare with others the prominent role of erythropoietin convention neurogenesis in protecting neurons (neurogenesis) and acts as a neurotropic factor in the central nervous system was obvious. The function of erythropoietin for the treatment of diseases associated with neuronal death makes it a suitable.

Several mechanisms have been proposed for the role of EPO with the results obtained in our plan is approved And the main cause of erythropoietin treatment response to its effect on neuronal and vascular structures (11-12-13).

Erythropoietin important role in the CNS of mice devoid of the EPO-R study found a lack of EPO-R. In these mice, large amounts of apoptosis and neuronal precursor cells showed reduction (14).

Erythropoietin may reduce endothelial cells lining the passage of leukocytes as well as 
increased resistance to endothelial cells against ischemia (17-18-19-20).

Totally in our study in two groups there were 16 patients with complete spinal cord injury and 44 patients with incomplete spinal cord injury there. In 4 days after the start of the study period, 16 patients with complete spinal cord injury no improvement was seen. Also in patients with incomplete spinal cord injury 13 of 21 patients (62\%) in case group and 2 of 23 (9\%) patients in control group were cured.

In the period of 6 months after the study, 2 of 8 patients $(25 \%)$ in case group with complete spinal cord injury were recovered. But no improvement was observed in the control group. Also in patients with incomplete spinal cord injury 12 of 19 patients (63\%) in case group and 5 of 21 patients (23\%) in the control group were cured.

As it is obvious in our study group erythropoietin significant progress Frankel classification of patients, respectively was seen.

\section{Conclusion}

The results of statistical analysis showed that intravenous erythropoietin improves the sensory and motor function in patients with acute spinal cord injury was associated with significant side effects were not recorded throughout the study.

Based on the results obtained, further improvement in patients receiving erythropoietin in the range of Frankel $C$ and $B$ in 4 days and 6 months after the trauma occurs; this difference is statistically significant from control group

It seems that erythropoietin decreases acute phase proteins, which has led to an improvement in sensory and muscle strength in patients with acute spinal cord injury.

\section{Suggestions}

1- Based on the results of animal studies that show the positive effect of EPO on improving clinical performance and improvement at the cellular level - molecules. And the result of our study is to improve clinical performance on 4 days and 6 months after treatment was effective and safe as well as erythropoietin can be the spinal cord acute injury of the tried.

2- The number of the patients in our study was 60patients which depending on the type and duration of follow-up studies it is recommended to achieve the best results with a higher volume of patients continues. Increasing number of specimens that will evaluate the effect of erythropoietin therapy on functional recovery in patients with higher accuracy and statistical value should be done.

3- Recommended in future studies followed patients for a longer period of time should be evaluated for vascular events.

4- EPO used dose was 500 units per $\mathrm{kg}$ of patient weight. Recommended lower doses of drugs used in future studies and comparing the results is suggested. The purpose of this therapy is to achieve a lower cost.

\section{References}

1. Wilson JR, Cho N, Fehlings MG. (2014). Acute Traumatic Spinal Cord Injury: Epidemiology, Evaluation, and Management.Spine Surgery Basics. Springer. p. 399-409.

2. EbrahimEltoray.translate by Abbas Kashi.(1388).Raveshha and osoltebbinokhaii.Iran

3. VIVES MJ, GARFIN SR, ABITBOL JJ, Vaccaro AR. (2013). Timing of Surgery Following Spinal Cord Injury. Fractures of the Cervical, Thoracic, and Lumbar Spine, 62.

4. Hall ED, Springer JE. (2004). Neuroprotection and acute spinal cord injury: a reappraisal. NeuroRx, 1(1), 80-100. 
5. Fisher M. (2011). New approaches to neuroprotective drug development. Stroke, 42(1 suppl 1), S24-S27.

6. H.RICHARD WINN,MD.professor and director of neurosurgery .YOUMANS NEUROLOGICAL SURGERY.SIXTH EDITION.

7. DavarAltafi, Mahdi Farhoodi. Therapeutic effects of intravenous erythropoietin in improving function outcome of intacerebral hemorrhage paitients , Tabriz university of medical science, faculty of medicine Tabriz, Iran,2010,

8. Shah RR, Tisherman SA. (2014). Spinal cord injury.Imaging the ICU Patient.Springer. p. 377-80.

9. Westgren N, Levi R. (1998). Quality of life and traumatic spinal cord injury.Archives of physical medicine and rehabilitation, 79(11), 1433-9.

10. Firth AM, Haldane SL. (1999 Mar 1). Development of a scale to evaluate postoperative pain in dogs. J Am Vet Med Assoc, 214(5), 651-9.

11. JelkmannW,Hellwing-Burgel T.2001 Biology of erythropoietin. AdvExp Med Biol 502:169-187.

34. Kobayashi T,YansaH,IwanagaT,Sasa;IR,Nagao M.2002 Epididymis is a novel site of erythropoietin production in mouse reproductive organs.BiochemBiophys Res Commun 296:145-151.

12. Patel NS,SharplesEJ,CuzzocreaS,ChatterjeePK,BrittiD,YaqoobMM,Theimermann C.2004Pretreatment with EPO reduses the injury and dysfunction caused by ischemia/reperfusion in the mouse kidney in vivo.Kidney In 66:983-989.

13. Brines M,Cerami A.2005 Emerginig biological roles for erythropoietin in the nervous system.Nat Rev Neurosci 6:484-494.

14. Dame C,JuulSE,Christensen RD.2001 The biology of erythropoietin in the central nervous system and its neurotrophic and neuroprotectivepotential.Biol Neonate 79:228-235.

15. FANG Xiang - qian,FAN Shun - wu,GUChuan - long. protection of erythropoietin on experimental spinal cord injury by reducing the expression of thrombospondin 6 - and transfprming growth factor- beta, . , department of orthopaedics sir runshau hospital, Zhejiany university, Hang zhau, China 2009 , jula .

16. Arishima, yoshiya MD, seyoguchi Takao MD, Yamoaura, Ichiro MD, Yone,KazunoriMD, Komya, setsuro MD. preventive effect of Erythropoietin on spinal cord cell apoptosis following acute tramatic injury in rats., 2006 oct.

17. SeyfriedDM,HanY,YangD,DingJ,Chopp M.2004 Erythropoietin promotes neurological recovery after intracerebral hemorrhage in rats.Int J Stroke.Auge :4(4):250-6.

18. Witko-SarsatV,RieuP,Descamps-LatschaB,LesavraP,Halbwachs-Mecarelli L.2000 Neutrophils:molecules, functions and pathophysiological aspects.Lab Invest 80:617-653.

19. Banks WA,JumbeNL,FarrellCL,NiehoffML,Heatherington AC.2004 Passage of erythropoietin ahents across the blood- brain barrier:a comparison of human and murine erythropoietin and the analog darbepoetinalfa. Eur J Pharmacol 505:93-10.

20. RecnyMA,ScobleHA,Kim Y.1987 Structural characterization of natural human urinary and recombinant DNAderived erythropoietin identification of des-arginine 166 erythropoietin .J BioChem 262:17156-17163.

21. Lipton P.1999 Ischemic cell death in the brain neurons.Physiol Rev 79:1431-1568.

22. Digicayliohlu M, BichetS,MartiHH,WengerRH,RivasLA,BauerC,Gassmann M.1995 Localization of specific erythropoietin binding sites in defined areas of the mouse brain.ProcNatlAcadSciUsa 92:3717-3720.

23. AnagnostouA,LeeES,KessimianN,LevinsonR,Steiner M.2006erythropoietin has a mitogenic and positive chemotactic effect on endothelial cells.ProcNatlAcadSci USA 87:5978-5982.

24. Martinez-Estrada OM,Rodriguez-MillanE,Gonzalez-De Vicente E,ReinaM,VIlaroS,Fabre M.2..3 Erythropoietin protects the in vivo blood brain barrier against VEGF-induced permability.Eur J Neurosci 18:2538-2544. 